\section{Validade de peso, altura e índice de massa corporal autorreferidos na população adulta de Brasília}

\section{Validity of self-reported height, weight and body mass index in the adult population of Brasilia, Brazil}

\author{
Priscilla Marcondelli Dias Thomaz' \\ Eduardo Freitas da Silva" \\ Teresa Helena Macedo da Costa"' \\ ' Pós-graduação em Ciências da Saúde da Universidade de Brasília, UnB. \\ " Departamento de Estatística da Universidade de Brasília, UnB. \\ II' Departamento de Nutrição da Universidade de Brasília.
}

Financiamento: Os recursos para execução da pesquisa foram financiados pelo CNPq (Processo 474665/04-6). Os autores declaram ausência de conflitos de interesse.

Correspondência: Priscilla Marcondelli Dias Thomaz. SQSW 306, Bloco A, Apto. 108 - Setor Sudoeste, 70673-431 Brasília, DF. E-mail: priscilla.nutricionista@gmail.com

\section{Resumo}

Objetivo: Avaliar a validade do peso, altura e índice de massa corporal (IMC) autorreferidos em adultos de Brasília e identificar os fatores associados às diferenças entre os valores medidos e autorreferidos. Métodos: Em amostra por conglomerados de 250 domicílios foram aferidos peso e altura de todos os adultos residentes, após entrevista. A concordância entre os parâmetros medidos e autorreferidos foi feita por Bland \& Altman (B\&A). Utilizou-se análise de variância múltipla e estimou-se a sensibilidade e especificidade para o excesso de peso (IMC $\geq 25 \mathrm{~kg} / \mathrm{m}^{2}$ ), estratificado por anos de estudo e faixa etária. Resultados: As entrevistas e mensurações foram feitas em 469 indivíduos. Os resultados de B\&A mostram viés (diferença dos valores medidos e autorreferidos) não significativo para o peso $(-0,17 \mathrm{~kg}, \mathrm{p}=0,1)$ e significativo para a altura $(-1 \mathrm{~cm}, \mathrm{p}<0,001)$. Os limites de concordância (LC) foram amplos: de $\pm 4 \mathrm{~kg}$ e de $-6 \mathrm{~cm}$ a $4 \mathrm{~cm}$. O viés do IMC foi de $-0,06 \mathrm{~kg} / \mathrm{m}^{2}$ e não significativo $(\mathrm{p}=0,08)$ e os LC de $1,5 \mathrm{a}+1,4 \mathrm{~kg} / \mathrm{m}^{2}$. Observou-se que homens com IMC $<25 \mathrm{~kg} / \mathrm{m}^{2}$ superestimam o peso, enquanto aqueles com sobrepeso e obesos o subestimam $(\mathrm{p}<0,01)$. Homens com +60 a de idade $(\mathrm{p}=0,037)$ e mulheres com $<12$ a de estudo $(\mathrm{p}<0,01)$ superestimam a altura. A sensibilidade e a especificidade para o excesso de peso foram para homens e mulheres, respectivamente: $94 \%$ e $88 \%$; $90 \%$ e $98 \%$. A sensibilidade foi de $77 \%$ para mulheres acima de 60 anos, de $75 \%$ para mulheres com 9 a 11 anos de estudo, e a especificidade de $78 \%$ para homens entre 30 e 39 anos. Conclusão: Em Brasília, a altura autorreferida pode ser utilizada para subgrupos de homens < 60anos de idade e mulheres com +12 anos de estudo. Homens com IMC adequado superestimam o peso corporal quando comparados com aqueles com sobrepeso ou obesos. As medidas autorreferidas prestam-se para acompanhamento populacional do excesso de peso.

Palavras-chave: Peso corporal. Altura corporal. Índice de massa corporal. Excesso de peso. Epidemiologia. Antropometria. 


\section{Abstract}

Objective: To assess the validity of self-reported weight, height and body mass index (BMI) estimates from adults in Brasilia and to identify factors associated to differences between measured and self-reported values. Methods: In a cluster sampling 250 household's were selected and weight and height were measured in all adults after an interview. Bland \& Altman (B\&A) was used to determined agreement between measured and self-reported parameters. The multiple analysis of variance was used to determine the associated factors. Sensitivity and specificity were used for excess weight (BMI $\geq$ $25 \mathrm{~kg} / \mathrm{m}^{2}$ ). Results: Interviews and measurements were done in 469 individuals. B\&A showed bias (difference between measured and self-reported values) was not significant for weight $(-0.17 \mathrm{~kg}, \mathrm{p}=0.1)$ and significant for height $(-1 \mathrm{~cm}, \mathrm{p}<0.001)$. The limits of agreement (LA) were wide: from $\pm 4 \mathrm{~kg}$ for weight and $-6 \mathrm{~cm}$ to $4 \mathrm{~cm}$ for height. BMI presented a non significant bias of $-0.06 \mathrm{~kg} /$ $\mathrm{m}^{2}(\mathrm{p}=0.08)$ and LA of -1.5 to $+1.4 \mathrm{~kg} / \mathrm{m}^{2}$. Men with a BMI $<25 \mathrm{~kg} / \mathrm{m}^{2}$ overestimated weight, while overweight or obese men underestimated it $(\mathrm{p}<0.01)$. Men older than $60 \mathrm{y}$ of age $(\mathrm{p}=0.037)$ and women with less than $12 \mathrm{y}$ of study $(\mathrm{p}<0.01)$ overestimated height. The sensitivity and specificity to detect excess weight were for men and women, respectively: $94 \%, 88 \%$ and $90 \%$, $98 \%$. Sensitivity to detect excess weight was $77 \%$ for 60 or over years old women and $75 \%$ for women between 9 to 11 years of study, while. specificity was $78 \%$ for men between 30 e 39 years old. Conclusion: In Brasilia, self-reported height can be used for subgroups of women with more than 12y of study and men under 60y of age. Men with adequate BMI over estimate their weight when compared to overweight and obese men. Self-reported measures can be used for excess weight population follow-up.

Keywords: Body weight. Body height. Body mass índex. Excess weight. Epidemiology. Anthropometry.

\section{Introdução}

O peso e a altura são as duas medidas antropométricas usuais na prática clinica e nas pesquisas. Estas medidas são obtidas com equipamentos específicos e amplamente disponíveis. Nesse contexto, os valores autorreferidos são uma forma simples, de baixo custo e que se aplica a grandes grupos populacionais ${ }^{1,2}$. Entretanto, discute-se na literatura a validade dos dados antropométricos autorreferidos.

$\mathrm{O}$ peso e a altura referidos apresentam alta correlação com o medido, o que representa uma alternativa interessante para medir e monitorar a prevalência da obesidade $^{3,4}$. No entanto, mesmo com este dado positivo, a revisão sistemática de Gorber et al. ${ }^{5}$ (2007), que analisou 64 pesquisas comparando peso, altura e IMC medidos com os autorreferidos, recomenda que o método seja utilizado com cautela, pois existem variáveis como sexo, idade e classificação socioeconômica, que atuam como vieses de aferição ${ }^{3,4,6}$. Apesar da alta correlação entre as informações obtidas pelos dois métodos, há uma tendência para a subestimação do peso e a superestimação da altura autorreferidos $^{5,7,8}$. Isso caracteriza ausência de concordância entre as medidas ${ }^{9}$.

Em relação à influência do sexo nos resultados do peso e da altura autorreferidos, observa-se subestimação do peso mais frequente entre as mulheres e superestimação da estatura mais frequente entre os homens ${ }^{2,10,11}$.

Kuczmarski et al. ${ }^{3}$ (2001) concluíram que o método de peso e altura autorreferidos é válido para jovens adultos, mas não para idosos acima de 60 anos, pois este último grupo tende a superestimar a altura. A estimação incorreta do peso e da altura conduz à estimação errônea do IMC, o que causaria influência direta na estimativa de prevalência de sobrepeso e obesidade. Por isso, várias pesquisas têm analisado a validade do IMC resultante do peso e da altura autorreferidos ${ }^{4,5,13,14}$.

Deduz-se daí a importância de pesquisas para a identificação dos fatores que 
influenciam os resultados do IMC baseado nos valores de peso e altura autorreferidos. Pesquisas anteriores foram realizadas com populações de outras regiões do Brasil, como Porto Alegre, Pelotas, Goiânia e Rio de Janeiro ${ }^{4,10,13,15}$. Contudo, não foram encontrados na literatura estudos que avaliam o peso e a altura referentes à população adulta de Brasília.

Nesse contexto, o objetivo deste estudo é avaliar a validade da estimativa de peso e altura autorreferidos e do IMC resultante na população adulta de Brasília, e identificar os fatores que influenciam os valores medidos e os referidos.

\section{Metodologia}

A população incluída no estudo foi composta por moradores de Brasília, Região Administrativa I, de acordo com a divisão dos Distritos Sanitários adotada pela Secretaria de Saúde do Distrito Federal ${ }^{16}$.

A amostragem foi estratificada com 4 estratos, com alocação proporcional ao tamanho do estrato. Foram usadas as fórmulas de Cochran ${ }^{17}$ (1977) para a estimação de proporções, e a precisão planejada foi de 95\%. A amostra foi obtida a partir da listagem dos domicílios cadastrados na Companhia Energética de Brasília (CEB) (totalizando 82.680 domicílios). A cobertura do cadastro desses endereços em Brasília é de 100\%. A unidade primária de amostragem foi a residência. Uma lista com todas as residências foi estratificada pelas quatro regiões de Brasília. De cada região foi obtida uma amostra aleatória simples de residências, os indivíduos foram localizados após sorteio de domicílios, e todos os adultos foram entrevistados. Para manter o tamanho amostral delineado na pesquisa, a cada endereço descartado outro foi arrolado seguindo a listagem fornecida pela CEB em fevereiro de 2005. O critério de reposição foi previsto no número de endereços sorteados inicialmente, onde cada conglomerado foi incluído com uma margem de $20 \%$ a mais de endereços.

Nestes termos, o número de domicílios sorteados totalizou 250 endereços, mantendo-se a proporcionalidade em cada uma das quatro regiões de Brasília: Asa Sul (113 domicílios, 45\%), Asa Norte (117 domicílios, 47\%), Vila Planalto (10 domicílios, 4\%) e Setor Militar Urbano (10 domicílios, 4\%).

Em cada domicílio os moradores com idade igual ou superior a 20 anos foram entrevistados no período de março de 2005 a novembro de 2006. Assumindo-se que cada domicílio tenha pelo menos dois adultos com 20 anos ou mais, o número estimado de indivíduos da amostra é 500 .

O projeto foi aprovado pelo Comitê de Ética da Faculdade de Ciências da Saúde da Universidade de Brasília (Proc. 101/2004) e todos os participantes assinaram o termo de consentimento livre e esclarecido.

$\mathrm{O}$ instrumento de pesquisa foi um questionário baseado no Questionário Internacional de Atividade Física (IPAQ), contendo perguntas sobre atividade física, identificação pessoal, dados demográficos e socioeconômicos, além do registro dos dados antropométricos ${ }^{18}$.

Foram utilizadas três balanças digitais com capacidade de 150 quilos e precisão de 100 gramas (Plenna, São Paulo, Brasil) e três estadiômetros portáteis (Alturexata, Belo Horizonte, Brasil), com 2,13 metros e precisão de 0,1 centímetro.

Foram realizados cinco encontros com a equipe de avaliadores no período da coleta para avaliar a reprodutibilidade entre os equipamentos de antropometria (balanças e estadiômetros) e os avaliadores. Em relação aos equipamentos, não houve diferença significativa na análise de variância entre as balanças $(p=0,14)$ e entre os estadiômetros $(\mathrm{p}=0,12)$. Medidas de peso e altura em seis indivíduos foram obtidas com os equipamentos, e o coeficiente de correlação intra classe (ICC) obtido foi igual a 1,00 com IC $95 \%$ de $0,98-1,00$.

$\mathrm{Na}$ avaliação da reprodutibilidade entre os avaliadores não foi observada diferença estatisticamente significativa na análise de variância $(\mathrm{p}=0,22)$. O ICC foi também utilizado para se avaliar as medidas realizada pelos avaliadores em seis indivíduos, com correlação de 1,00 (IC 95\% 0,99 - 1,00). 
No domicílio, após assinatura do termo de consentimento, os entrevistadores aplicavam o questionário. Ao final da entrevista era solicitado ao indivíduo informar seu peso e altura. Somente depois de respondida esta pergunta eram aferidos o peso e a altura. Todos os indivíduos foram medidos sem sapatos e trajando roupas leves. Para os poucos indivíduos que não usavam roupas leves utilizou-se como critério de ajuste a redução de 500g no momento do registro do peso aferido. $\mathrm{O}$ valor de $500 \mathrm{~g}$ corresponde ao peso de camisa de malha básica de algodão e calça jeans.

A metodologia utilizada para a mensuração de peso e altura foi aquela descrita pela Organização Mundial de Saúde ${ }^{19}$. A balança foi posicionada em superfície plana e firme. $\mathrm{O}$ individuo foi orientado a retirar todos os adornos e trajar roupas leves (camiseta, shorts ou saia de tecido leve). A balança foi ligada pelo avaliador. Quando a marcação do zero estava no visor o voluntário foi orientado a pisar descalço com um pé em cada lado da plataforma da balança, permanecer parado em pé, com braços ao longo do corpo, face para frente e o olhar para o horizonte. O peso em quilogramas foi registrado no formulário e o indivíduo orientado a descer da balança. $\mathrm{O}$ peso medido foi informado ao voluntário. O estadiômetro Alturexata (Belo Horizonte, Brasil) foi montado no domicílio. O estadiômetro é independente de suporte de parede e possui régua com haste metálica horizontal para tocar o topo da cabeça, e tablado com apoio traseiro para encostar o calcanhar. O voluntário foi instruído a tirar todos os adornos de cabelo e cabeça, pisar descalço na plataforma do estadiômetro, de frente para o avaliador. Foi posicionado com os pés unidos, calcanhares tocando a placa traseira, joelhos estendidos, olhar voltado para o horizonte. A cabeça do voluntário foi posicionada de modo que os olhos estivessem no mesmo nível das orelhas. A haste metálica horizontal foi arrastada gentilmente até o topo da cabeça do voluntário, que foi orientado a fazer uma inspiração, reter $\mathrm{o}$ ar por alguns segundos e se manter bem parado e ereto. A altura foi lida no ponto exato da mensuração, o voluntário orientado a descer do estadiômetro e o valor em centímetros foi anotado no formulário. $\mathrm{O}$ valor medido foi informado ao voluntário.

Utilizou-se a classificação do IMC recomendada pela OMS com os valores de < $18,5 \mathrm{~kg} / \mathrm{m}^{2}$ para baixo peso; $18,5 \mathrm{a} 24,99 \mathrm{~kg} /$ $\mathrm{m}^{2}$ para a faixa de normalidade; 25,0 a 29,99 $\mathrm{kg} / \mathrm{m}^{2}$ para sobrepeso e IMC $\geq 30,0 \mathrm{~kg} / \mathrm{m}^{2}$ para obesidade ${ }^{20}$.

As atividades físicas foram analisadas pelo tempo, de acordo com a recomendação de 150 minutos de atividade por semana ${ }^{21}$. O tempo de atividade física semanal foi classificado somando-se os minutos semanais despendidos em caminhadas com os minutos semanais empregados em outras atividades físicas moderadas, mais os minutos por semana em atividades físicas vigorosas com fator multiplicador 2. Segundo esta forma de análise, os entrevistados foram classificados em quatro diferentes níveis de atividade física, de acordo com o escore obtido em minutos: 0, inativo; 1 - 149, insuficientemente ativo; 150 - 499, ativo; >= 500 , muito ativo ${ }^{22}$.

Para a avaliação do poder aquisitivo, utilizou-se o Critério de Classificação Econômica Brasil $^{23}$, desenvolvido pela Associação Brasileira de Anunciantes, pela Associação Nacional de Empresas de Pesquisa de Mercado e pela Associação Brasileira de Institutos de Pesquisa de Mercado.

\section{Análise Estatística}

A validade do peso e da altura autorreferidos foi realizada separadamente para cada sexo. Os métodos estatísticos para a análise incluíram parâmetros descritivos (média, desvio padrão), análise de variância múltipla, análise de sensibilidade e especificidade.

A concordância entre os valores de peso e altura medidos e referidos foi analisada com o método de Bland \& Altman (B\&A) . Também foi feita a mesma análise para o IMC calculado a partir dos dados de peso e altura medidos e autorreferidos. Foi 
determinado, a priori, pelos pesquisadores, o limite aceitável de variação de $\pm 2 \mathrm{~kg}$ para o peso e de $\pm 1 \mathrm{~cm}$ para a altura autorreferida. Esse limite aceitável foi estabelecido devido à variação de peso usual, à variação entre os equipamentos no qual os indivíduos obtêm os valores de suas medidas, e ao tempo entre a última medida efetiva e a participação na pesquisa. O limite aceitável de variação para o IMC foi estabelecido, a posteriori, em \pm $0,8 \mathrm{~kg} / \mathrm{m}^{2}$, baseado nos valores médios de peso e altura dos indivíduos. A definição dos valores de limite aceitáveis é importante para que eles sejam contrastados com os limites de concordância (média da diferença \pm 2 desvios padrões) obtidos pela análise de Bland \& Altman. Na analise de B\&A evidencia-se a tendência de comportamento das medidas através do coeficiente de correlação de Pearson.

Fatores demográficos, socioeconômicos e relacionados à saúde foram examinados como potenciais variáveis explicativas dos possíveis vieses para a diferença dos pesos e das alturas autorreferidos e medidos. Estes fatores incluíram idade, classe socioeconômica, estado civil, anos de estudo, escore de atividade física e IMC. As diferenças entre o peso medido e o autorreferido, e entre a altura medida e a autorreferida foram usadas como variáveis dependentes na análise de variância múltipla. O teste post-hoc de Tukey foi realizado para determinar as diferenças entre os estratos das variáveis da análise de variância múltipla.

A validade do peso e da altura autorreferidos para caracterizar excesso de peso foi analisada pelos indicadores de sensibilidade, especificidade, valores preditivos positivos e negativos. Análise de sensibilidade e especificidade para o excesso de peso foi também realizada para anos de estudo e faixas etárias. Considerou-se com excesso de peso as pessoas com IMC $\geq 25$, incluindo-se, portanto, os obesos nesse grupo.

Todas as informações foram tabuladas em planilha eletrônica (Excel, Microsoft Office 2000) e analisadas no SAS Versão 9.1.3. O nível de significância utilizado foi de $\mathrm{p}<0,05$.

\section{Resultados}

Duzentos e cinquenta endereços foram visitados, com uma amostra de 469 indivíduos entrevistados, sendo $57 \%$ do sexo feminino. Do total de endereços sorteados, houve a necessidade de se substituir 53\% devido a três motivos: residência sem morador, endereço comercial e recusa de todos os moradores em participar da pesquisa. A recusa a responder a pesquisa foi de $13 \%$ dos indivíduos. A amostra de 469 indivíduos correspondeu a $94 \%$ do total esperado.

A média de idade foi de 44 anos (DP 16), variando de 20 a 91 anos. Na classificação socioeconômica, $44 \%$ dos indivíduos pertenciam ao grupo com nível mais alto (classe A), $37 \%$ à classe B e $19 \%$ foram classificados nos grupos com menores renda e instrução do chefe da família (classes C, D e E). A média de anos de estudo foi de 13, com variação entre 9 e 17 anos e mediana de 15 anos. A média de peso dos homens foi de 78,5 kg (DP 13,7) e a altura foi de $1,73 \mathrm{~m}$ (DP 0,07). Para as mulheres a média de peso foi de $62,8 \mathrm{~kg}$ (DP 12,5) e de 1,59 m (DP 0,06) de altura.

Em relação ao índice de massa corporal (IMC), uma vez que somente $1 \%$ apresentou baixo peso, essas pessoas foram inseridas na faixa de IMC adequado. Assim constatou-se que $52 \%$ dos indivíduos encontravam-se com IMC dentro do normal, 33\% com sobrepeso e $15 \%$ eram obesos (Tabela 1).

\section{Análise multivariada}

Foi realizada uma análise de variância múltipla para identificar os fatores associados às diferenças médias entre peso e altura medidos e autorreferidos por sexo (Tabela 2). Para as mulheres, a diferença entre o peso medido e o autorreferido não apresentou associação com nenhum dos parâmetros incluídos no modelo $(p=0,36)$. Os homens com IMC abaixo de $25 \mathrm{~kg} / \mathrm{m}^{2}$ tendem a superestimar o próprio peso, enquanto aqueles com sobrepeso e obesos tendem a subestimá-lo $(\mathrm{p}<0,01)$.

Em relação à altura, as variáveis consideradas como vieses foram a idade no sexo 
Tabela 1 - Características de adultos de Brasília separados por sexo. Brasília, 2006-2007.

Table 1 - Characteristics according to sex of adults from Brasilia, Brazil, 2006-2007.

\begin{tabular}{|c|c|c|c|c|}
\hline & \multicolumn{2}{|c|}{$\begin{array}{l}\text { Masculino } \\
(n=203)\end{array}$} & \multicolumn{2}{|c|}{$\begin{array}{l}\text { Feminino } \\
(\mathrm{n}=206)\end{array}$} \\
\hline & $\mathrm{n}$ & $\%$ & $\mathrm{n}$ & $\%$ \\
\hline \multicolumn{5}{|l|}{ Idade (anos) } \\
\hline $20-29$ & 45 & 22,2 & 60 & 22,6 \\
\hline $30-39$ & 41 & 20,2 & 54 & $2-, 3$ \\
\hline $40-49$ & 46 & 22,7 & 66 & 24,8 \\
\hline $50-59$ & 39 & 19,2 & 41 & ‘5,4 \\
\hline 60 ou mais & 32 & 15,8 & 45 & 16,9 \\
\hline \multicolumn{5}{|l|}{ Nível socioeconômico } \\
\hline A & 90 & 44,3 & 114 & 42,9 \\
\hline B & 80 & 39,4 & 94 & 35,3 \\
\hline $\mathrm{C}+\mathrm{D}+\mathrm{E}$ & 33 & 16,3 & 58 & 21,8 \\
\hline \multicolumn{5}{|l|}{ Anos de estudo } \\
\hline $0-8$ anos & 17 & 8,4 & 42 & 15,8 \\
\hline $9-11$ anos & 63 & 31,0 & 99 & 37,2 \\
\hline $12-15$ anos & 48 & 23,6 & 61 & 22,9 \\
\hline 16 ou mais anos & 75 & 36,9 & 64 & 24,1 \\
\hline \multicolumn{5}{|l|}{ Estado civil } \\
\hline Solteiro & 60 & 29,6 & 83 & 31,2 \\
\hline Casado & 128 & 63,0 & 137 & 51,5 \\
\hline Divorciado + viúvo & 15 & 7,4 & 46 & 17,3 \\
\hline \multicolumn{5}{|c|}{$\begin{array}{l}\text { Escore atividade ( } 150 \mathrm{~min} \text {. de atividade } \\
\text { física/semana) }\end{array}$} \\
\hline Inativo & 45 & 22,2 & 56 & 21,0 \\
\hline Pouco ativo & 43 & 21,2 & 79 & 29,7 \\
\hline Ativo & 75 & 36,9 & 92 & 34,6 \\
\hline Muito ativo & 40 & 19,7 & 39 & 14,7 \\
\hline \multicolumn{5}{|c|}{ IMC (Índice de Massa Corporal) medido } \\
\hline Baixo Peso + Eutrófico & 83 & 40,9 & 160 & 60,1 \\
\hline Sobrepeso & 88 & 43,3 & 68 & 25,6 \\
\hline Obeso & 32 & 15,8 & 38 & 14,3 \\
\hline
\end{tabular}

masculino e os anos de estudo no feminino, sendo que os homens com mais de 60 anos de idade $(\mathrm{p}=0,037)$ e as mulheres com menos de 12 anos de estudo $(\mathrm{p}<0,01)$ tendem a superestimar a própria altura.

\section{Análise de Bland \& Altman}

Observou-se diferença sistemática entre as duas mensurações (viés) com média igual $\mathrm{a}-0,17 \mathrm{~kg}$ para o peso $(\mathrm{p}=0,10)$ e de $-1 \mathrm{~cm}$ $(\mathrm{p}<0001)$ para a altura (Figura 1). Ou seja, o peso medido é, em média, muito próximo ao peso referido, e a altura se encontra, na média, significativamente inferior à altura referida. O limite de concordância (média da diferença de 2 desvios padrões) para o peso corporal foi de $\pm 4 \mathrm{~kg}$ e alguns pontos apresentaram-se discrepantes (15 de 469, $3,2 \%)$. Da mesma forma, para a altura, a maioria dos pontos encontra-se dentro dos limites de concordância, entre $-6 \mathrm{~cm} \mathrm{e}+4$ cm e, neste caso, cerca de $3,8 \%$ dos pontos apresentam discrepância entre os valores (18 de 469 pontos). Consequentemente, o valor do IMC medido está, em média, muito próximo e não difere significativamente do referido, com a maioria dos pontos localizada dentro dos limites de concordância ($\left.1,5 \mathrm{a}+1,4 \mathrm{~kg} / \mathrm{m}^{2}\right)$ com a média da diferença igual a $-0,06 \mathrm{~kg} / \mathrm{m}^{2}(\mathrm{p}=0,08)$ (Figura 1). Não se verificou diferença na distribuição 
Tabela 2 - Diferenças de medias entre valores de peso e altura medidos e autorreferidos para os fatores socioeconômicos de homens e mulheres. Brasília, 2006-2007.

Table 2 - Mean difference of measured and self-reported weight and height values for socioeconomic factors of men and women. Brasilia, Brazil, 2006-2007.

\begin{tabular}{|c|c|c|c|c|c|c|}
\hline & \multicolumn{3}{|c|}{ Masculino $(n=203)$} & \multicolumn{3}{|c|}{ Feminino $(n=266)$} \\
\hline & $\mathrm{N}$ & $\begin{array}{l}\text { Diferença } \\
\text { Média } \\
\text { Peso }(k g) \\
\end{array}$ & $\begin{array}{l}\text { Diferença } \\
\text { Média } \\
\text { Altura }(\mathrm{cm})\end{array}$ & N & $\begin{array}{l}\text { Diferença } \\
\text { Média Peso } \\
(\mathrm{kg})\end{array}$ & $\begin{array}{c}\text { Diferença } \\
\text { Média } \\
\text { Altura }(\mathrm{cm})\end{array}$ \\
\hline \multicolumn{7}{|l|}{ Faixa Etária (anos) } \\
\hline $19-29$ & 45 & $-0,73$ & $-0,38 a b$ & 60 & $-0,68$ & $-1,01$ \\
\hline $30-39$ & 41 & $-0,39$ & $-0,77 a b$ & 54 & $-0,16$ & $-0,92$ \\
\hline $40-49$ & 46 & $-0,21$ & $-0,12 \mathrm{a}$ & 66 & $-0,10$ & $-1,14$ \\
\hline $50-59$ & 39 & $-0,04$ & $-1,05 a b$ & 41 & 0,50 & $-1,15$ \\
\hline \multirow{2}{*}{60 ou mais } & 42 & -1.14 & $-1,88 b$ & 45 & $-0,08$ & $-1,86$ \\
\hline & & NS & $* *$ & & NS & NS \\
\hline \multicolumn{7}{|c|}{ Nível de Classe Econômica } \\
\hline A & 90 & $-0,05$ & $-0,94$ & 114 & 0,12 & $-0,90$ \\
\hline B & 80 & $-0,47$ & $-0,55$ & 94 & $-0,10$ & $-1,26$ \\
\hline \multirow[t]{2}{*}{$C+D+E$} & 33 & $-0,3$ & $-0,81$ & 58 & $-0,11$ & $-1,65$ \\
\hline & & NS & NS & & NS & NS \\
\hline \multicolumn{7}{|l|}{ Estado Civil } \\
\hline Casado & 128 & $-0,41$ & $-0,73$ & 137 & $-0,09$ & $-1,12$ \\
\hline Divorciado + Viúvo & 15 & $-0,44$ & $-1,22$ & 46 & 0,11 & $-1,26$ \\
\hline \multirow[t]{2}{*}{ Solteiro } & 60 & $-0,11$ & $-0,72$ & 83 & 0,06 & $-1,27$ \\
\hline & & NS & NS & & NS & NS \\
\hline \multicolumn{7}{|l|}{ Nível de Anos de Estudo } \\
\hline $0-8$ anos & 17 & $-0,42$ & $-1,26$ & 42 & 0,10 & $-2,47 b$ \\
\hline $9-11$ anos & 63 & $-0,14$ & $-0,41$ & 99 & $-0,01$ & $-1,25 a b$ \\
\hline $12-15$ anos & 48 & $-0,26$ & $-0,44$ & 61 & 0,06 & $-0,61 a$ \\
\hline \multirow[t]{2}{*}{16 ou mais anos } & 75 & $-0,32$ & $-1,15$ & 64 & $-0,14$ & $-0,81 a$ \\
\hline & & NS & NS & & NS & $* * *$ \\
\hline \multicolumn{7}{|l|}{ Escore de AF } \\
\hline Ativo & 75 & $-0,38$ & $-1,11$ & 92 & 0,14 & $-1,38$ \\
\hline Inativo & 45 & 0,20 & $-0,67$ & 56 & $-0,003$ & $-0,87$ \\
\hline Muito ativo & 40 & $-0,07$ & $-0,98$ & 39 & 0,54 & $-1,60$ \\
\hline \multirow[t]{2}{*}{ Pouco ativo } & 43 & $-0,70$ & $-0,06$ & 79 & $-0,45$ & $-0,99$ \\
\hline & & NS & NS & & NS & NS \\
\hline \multicolumn{7}{|l|}{ Nível de IMC medido } \\
\hline Baixo Peso + Normal & 83 & $-0,87 b$ & $-0,61$ & 160 & $-0,19$ & $-1,00$ \\
\hline Obeso & 32 & $0,14 a$ & $-1,15$ & 38 & $-0,06$ & $-1,57$ \\
\hline \multirow[t]{2}{*}{ Sobrepeso } & 88 & $0,17 a$ & $-0,77$ & 68 & 0,44 & $-1,41$ \\
\hline & & * & NS & & NS & NS \\
\hline
\end{tabular}

Análise de variância múltipla: ${ }^{*} \mathrm{P}=0,0028 * * \mathrm{P}=0,0374 * * * \mathrm{P}=0,0093 \mathrm{NS}=$ não significativo IMC $=$ Índice de Massa Corporal Multiple variance analysis: ${ }^{*} P=0,0028 * * P=0,0374 * * * P=0,0093$ NS $=$ non significant $B M I=$ Body Mass Index Os valores com subescritos distintos apresentam diferença pela teste post-hoc de Tukey.

Values with distinct subscript are different by the Tukey post-hoc test.

de pontos discrepantes (fora dos limites de concordância) entre os sexos. Assim, em função das diferenças estatísticas encontradas para os vieses existe concordância para as medidas de peso e IMC, mas não para a altura. A distribuição dos indivíduos no gráfico para o IMC mostra maior concentração de mulheres com IMC próximo a 20 e uma dispersão maior do IMC feminino em relação ao masculino (Figura 1). Os limites de concordância foram mais amplos para peso, altura e IMC do que os limites 

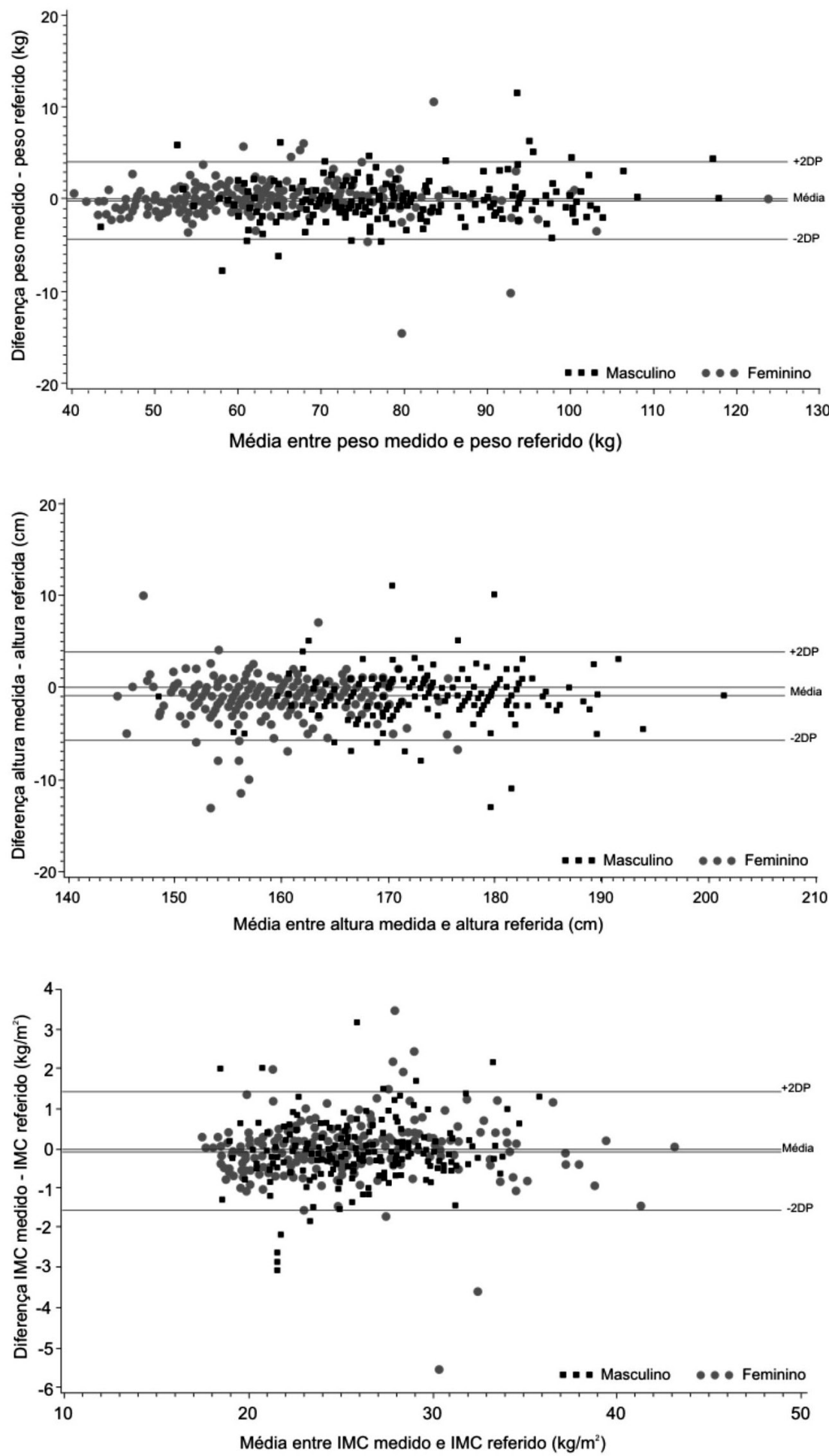

Figura 1 - Gráficos de Bland e Altman mostrando as diferenças médias e os limites de concordância de $95 \%$ para o peso, a altura e o índice de massa corporal (IMC) medidos e referidos na população adulta de Brasília, 2006-2007.

Figure 1 - Bland \& Altman plots showing mean difference and 95\% limits of agreement for measured and self-reported weight, height and body mass index (BMI) for adults from Brasilia, Brazil, 2006-2007. 
aceitáveis de variação estabelecidos para essas medidas (Figura 1). Não houve correlação significativa entre os valores médios e as médias das diferenças (vieses) para altura em ambos os sexos (homens $r=0,016 ; p=$ 0,81 e mulheres $r=-0,016 ; p=0,79$ ), o que possibilita o uso do viés como um fator de correção. Também não ocorreu correlação entre média e vieses de peso e IMC para as mulheres (peso $\mathrm{r}=0,041, \mathrm{p}=0,51$ e IMC $\mathrm{r}=$ $0,03 ; p=0,64)$. Para os homens a correlação da média com os vieses de peso e IMC foram positivas e significativas (peso $\mathrm{r}=0,17 ; \mathrm{p}=$ $0,01$ e IMC $r=0,17 ; p=0,01)$, confirmando a tendência evidenciada para o peso obtida na analise multivariada.

\section{Análise de Sensibilidade e Especificidade para o excesso de peso}

Os resultados referentes aos valores de sensibilidade foram de $94 \%$ para os homens e de $88 \%$ para as mulheres, o que corresponde à capacidade do indivíduo com excesso de peso de referir seu IMC corretamente. Em relação à especificidade, os valores também foram elevados, $90 \%$ para o sexo masculino e $98 \%$ para o feminino, o que representa o valor referente à capacidade do indivíduo sem excesso de peso de referi-lo corretamente (Tabela 3).

Em relação aos valores preditivos, 93\% dos homens e $97 \%$ das mulheres diagnosticadas com excesso de peso por meio das medidas autoreferidas relataram corretamente seus dados de peso e altura.

$O$ valor preditivo negativo se refere aos indivíduos sem excesso de peso. Portanto, encontramos também um resultado satisfatório, com $92 \%$ dos homens e $93 \%$ das mulheres tendo afirmado corretamente estar sem excesso de peso.

$\mathrm{Na}$ análise de sensibilidade e especificidade para o excesso de peso estratificado para anos de estudo, observou-se sensibilidade de $75 \%$ (IC 95\% de $62-90 \%$ ) para mulheres com 9 a 11 anos de estudo ( $\mathrm{n}=$ 99) e valor preditivo negativo de $87 \%$ (IC $95 \%$ de 79 - 95\%). Todos os outros valores de sensibilidade para homens e mulheres nas outras faixas de anos de estudo ficaram entre 91 e $100 \%$, e de especificidade entre 87 e 100\% (dados não apresentados).

$\mathrm{Na}$ análise estratificada para as faixas de idade entre homens e mulheres para o excesso de peso, observou-se entre homens de 30 a 39 anos $(\mathrm{n}=41)$ especificidade de $78 \%$ (IC 95\% de 60 - 98\%) e valor preditivo negativo de $83 \%$ (IC $95 \%$ de $65-100 \%$ ). A sensibilidade de homens de 30 a 39 anos foi de $86 \%$ (IC $95 \%$ de $71-100 \%$ ), e o valor preditivo positivo de $83 \%$ (IC 95\% de 66 - 99\%), confirmando que os homens dessa faixa de idade tendem a superestimar o seu excesso de peso. Observamos sensibilidade de $77 \%$ (IC 95\% de 60 - 94\%) para mulheres com 60 anos ou mais $(\mathrm{n}=45)$ e valor preditivo negativo de $76 \%$ (IC $95 \%$ de 58 - 93\%), enquanto a especificidade e valor preditivo positivo foram de $100 \%$ para ambos, confirmando que as mulheres nessa faixa etária tendem a subestimar o seu excesso de peso.

\section{Discussão}

A presente investigação demonstrou que, na população de Brasília, as médias de peso, altura e IMC calculadas com os valores autorreferidos aproximam-se dos aferidos. No entanto, deve-se considerar que os resultados mostram um limite de concordância muito amplo, o que pode levar a erro significativo na informação individual. $\mathrm{Na}$ análise de variância múltipla foi possível observar que existem características dos indivíduos que podem influenciar na validade dos resultados autorreferidos, tais como a idade e o IMC para os homens e a escolaridade para as mulheres.

De uma maneira geral, a população deste estudo tende a subestimar o peso (média de $-0,17 \mathrm{~kg}$ ) e a altura (média de $-1 \mathrm{~cm}$ ), resultados semelhantes aos encontrados por Schmidt et al. ${ }^{10}$ (1993) com 659 adultos de Porto Alegre (diferença entre peso referido e medido de - 0,06 kg). Em outro estudo realizado no Brasil, Fonseca et al. ${ }^{4}$ (2004) observaram uma tendência à superestimação dos dados com uma diferença média de 1,09 kg para o peso, e de 0,65 $\mathrm{cm}$ para a 
Tabela 3 - Prevalência de excesso de peso baseado nos valores medidos e referidos e nos valores testados para a prevalência de excesso de peso baseado nos valores autorreferidos. Table 3 - Excess weight prevalence based on measured and self-reported values and tested prevalence values for excess weight based on self-reported values.

\begin{tabular}{|c|c|c|c|c|}
\hline & $\begin{array}{l}\text { Homens } \\
(n-203)\end{array}$ & IC $95 \%$ & $\begin{array}{c}\text { Mulheres } \\
(n=266)\end{array}$ & IC 95\% \\
\hline Prevalência de Excesso de Peso & $\%$ & & $\%$ & \\
\hline Dados Mensurados & 59 & $52-65$ & 39 & $33-45$ \\
\hline Dados Autorreferidos & 59 & $52-66$ & 35 & $30-41$ \\
\hline Sensibilidade & 94 & $88-97$ & 88 & $80-94$ \\
\hline Especificidade & 91 & $82-95$ & 98 & $94-99$ \\
\hline Valor Preditivo positivo & 93 & $87-97$ & 97 & $90-99$ \\
\hline Valor Preditivo negativo & 92 & $83-96$ & 93 & $88-96$ \\
\hline
\end{tabular}

Excesso de peso definido pelo IMC (índice de massa corporal) $\geq 22,0, \mathrm{~kg} / \mathrm{m}^{2}$

Excess weight defined by $B M I$ (body mass index) $\geq 22,0, \mathrm{~kg} / \mathrm{m}^{2}$

IC - Intervalo de Confiança de 95\% / Cl-95\% Confidence Interval

altura em um estudo longitudinal com 3.713 funcionários públicos entre 22 e 70 anos.

Na Suécia, o estudo de Nyholm et al. ${ }^{14}$ (2007) verificou uma diferença média relativamente alta entre o peso medido e referido de 1,6 kg para os homens e 1,8 $\mathrm{kg}$ para as mulheres. Em relação à altura foi observada uma diferença média de $-0,3 \mathrm{~cm}$ nos sexo masculino e de - 0,4 cm no feminino.

No nosso estudo, a análise de variância múltipla mostrou que homens com IMC baixo e normal superestimam o peso e aqueles com sobrepeso e obesos o subestimam. Schmidt et al. ${ }^{10}$ (1993) e Peixoto et al. ${ }^{13}$ (2006) também encontraram resultados semelhantes. Estes padrões de comportamento podem ser explicados pela insatisfação com a imagem corporal quando comparados aos padrões de beleza culturalmente valorizados ${ }^{10,24}$.

Com relação às mulheres, nosso estudo não apresentou diferença significativa entre as informações de peso medido e referido (média da diferença de 0,005 kg). Isso mostra que as mulheres têm um bom conhecimento do seu peso corporal. O estudo de revisão de Engstrom et al. ${ }^{25}$ (2003) observou que as mulheres dos Estados Unidos, Grã Bretanha, Escandinávia, Europa Ocidental, Austrália, Nova Zelândia e Ásia apresentam uma tendência a relatar um peso menor que o real e uma altura maior que a referida em grande parte das pesquisas sobre o tema. A pesquisa incluiu 34 estudos sobre a precisão do peso autorreferido em 57.172 mulheres e todos observaram subestimação do peso, enquanto 26 estudos foram analisados sobre altura autorreferida em 39.244 mulheres e 21 constataram superestimação da altura.

Uma explicação para a diferença entre os resultados de Engstrom et al. ${ }^{25}$ (2003) e os encontrados nesse estudo pode ser o fato desta amostra ser basicamente constituída por mulheres com alto nível socioeconômico e educacional. Villanueva ${ }^{11}$ (2001) observou que, quanto maior o nível educacional, menor a diferença entre o peso medido e o autorreferido.

Para as mulheres, a altura sofreu influência da escolaridade, pois verificou-se associação de mulheres com menos de 12 anos de escolaridade com superestimação da própria altura. Brunner ${ }^{26}$ (2007) confirma esses achados em seu estudo realizado com 381 mulheres com média de 30 anos de idade. Nas mulheres com 9 a 11 anos de estudo obtivemos menor sensibilidade para referir o excesso de peso. Nesse grupo de escolaridade intermediária as pressões culturais que interferem no relato do excesso de peso parecem influenciar mais do que para mulheres com menor ou maior escolaridade.

Uma pesquisa realizada em Goiânia observou que a altura foi superestimada pelas mulheres, independentemente da faixa etária, escolaridade, renda, estatura e IMC. No entanto, houve um aumento da 
altura autorreferida para as mulheres com menos escolaridade, menor estatura, e classificações extremas de IMC, como aquelas que apresentam baixo peso ou obesidade ${ }^{13}$.

Com relação ao achado de superestimação da altura nos homens com mais de 60 anos de idade em nossa pesquisa, os dados encontrados são semelhantes a vários estudos. Esse fato poderia ser explicado pelo maior tempo que as pessoas mais velhas passam sem aferição antropométrica, associado ao processo natural da redução da altura com o aumento da idade ${ }^{2,3,27}$.

Com relação ao cálculo do IMC baseado nos valores de peso e altura auto-referidos, nosso estudo encontrou médias muito próximas entre o IMC medido e o referido para homens e mulheres, o que confirma, parcialmente, os achados de Silveira et al. ${ }^{15}(2005)$, cujos resultados mostraram-se precisos para as médias de IMC do sexo masculino.

Ao contrário dos achados de Hill e Roberts $^{12}$ (1998) em uma pesquisa com homens e mulheres de 16 a 64 anos de idade, nos quais a subestimação do IMC pode ser explicada tanto pela superestimação da altura quanto pela subestimação do peso. Esse fato foi confirmado no estudo de Niedhammer ${ }^{2}$ (2000), com subestimação do IMC $\left(0,29\right.$ e $0,44 \mathrm{~kg} / \mathrm{m}^{2}$, pelos homens e pelas mulheres, respectivamente), onde a altura foi superestimada em $0,38 \mathrm{~cm}$ pelos homens e em $0,40 \mathrm{~cm}$ pelas mulheres, e o peso foi subestimado em $0,54 \mathrm{~kg}$ pelos homens e em 0,85 $\mathrm{kg}$ pelas mulheres. Segundo a pesquisa de Silveira et al. ${ }^{15}$ (2005), as mulheres com mais de 50 anos e com baixa renda apresentaram resultados de subestimação de IMC em mais de $2 \mathrm{~kg} /$ $\mathrm{m}^{2}$. No nosso estudo, a sensibilidade foi de $77 \%$ para o excesso de peso referido entre mulheres com mais de sessenta anos de idade e o valor preditivo negativo acompanhou esse resultado, confirmando que as mulheres mais velhas tendem a subestimar o seu excesso de peso.

Os limites aceitáveis de variação do peso $( \pm 2 \mathrm{~kg})$ para a altura $( \pm 1 \mathrm{~cm})$ autorreferida foram preestabelecidos no nosso estudo a partir de nossa experiência com antropometria. Esses limites foram recentemente descritos por Brestoff et al. ${ }^{28}$ (2011) em estudo detalhando dos vieses encontrados nos resultados de peso, altura e IMC autorreferidos. Esses autores descrevem variação aceitável de $2 \mathrm{~kg}, 2 \mathrm{~cm}$ e $1,4 \mathrm{~kg} / \mathrm{m} 2$ atribuídos aos erros inerentes de mensuração e flutuações temporais das medidas. O viés médio global para o IMC, obtido por Brestoff et al., foi de $-1.34 \mathrm{~kg} / \mathrm{m}^{2}$ com valores de variação entre $-3,54 \mathrm{e}+3,01$ $\mathrm{kg} / \mathrm{m}^{2}$. Na população adulta de Brasília, o viés global e os limites concordância de 95\% apresentaram melhor concordância. Ainda no estudo de Brestoff et al. ${ }^{28}$ (2011), os erros que mais contribuem para o viés de relato do IMC são apresentados em ordem decrescente: sub-relato do peso combinado com super-relato da altura; sub-relato do peso com relato preciso da altura; e relato preciso do peso com super-relato da altura ${ }^{28}$. No nosso estudo, de acordo com o gráfico de Blend \& Altman, evidenciamos um relato mais preciso do peso e sub-relato da altura.

Estudos anteriores ${ }^{4,6}$ encontraram valores de especificidade em torno de $98 \%$ para os indivíduos classificados como obesos segundo a aferição. No nosso estudo, os resultados são para o excesso de peso e abrangem a população com sobrepeso e obesos, onde a especificidade é de $90 \%$ para o sexo masculino e de $98 \%$ para o feminino. A sensibilidade em estudos anteriores foi de $75 \%$, mas descritos para obesos e não para excesso de peso ${ }^{4,6}$. Osuna-Ramirez et al. ${ }^{29}$ (2006) mostram aumento dos valores de sensibilidade e especificidade quando os dados são agrupados para o excesso de peso do que aqueles descritos para o sobrepeso e obesidade separadamente. Os valores para o excesso de peso em adultos mexicanos foram de $82 \%$ e $91 \%$ para sensibilidade e especificidade, respectivamente ${ }^{29}$.

A partir destes resultados podemos afirmar que a utilização do peso e da altura autorreferidos para a prática clínica ou para pesquisas direcionadas à clínica deve ser evitada, pois, apesar de a diferença média dos dados referidos ser pequena, a discrepância individual foi clinicamente 
significante para os indivíduos ${ }^{25}$. Deve-se considerar a limitação desse estudo, que obteve uma taxa de recusa acima do desejável, ou seja, foi superior a $10 \%$. No entanto, devido às características do delineamento amostral, os indivíduos entrevistados representam proporcionalmente o universo dos setores que compõem a cidade de Brasília. Os resultados obtidos podem auxiliar na correção de dados autorreferidos da população, especialmente nos inquéritos conduzidos por telefone.

Concluindo, evidenciamos que o uso de dados autorreferidos para os adultos de Brasília é adequado para o acompanhamento da altura de mulheres adultas com mais de 12 anos de estudo e de homens adultos com menos de 60 anos de idade. O peso corporal autorreferido é superestimado por homens com IMC baixo e normal, quando comparados com homens com sobrepeso ou obesidade. As mulheres não apresentam associação dos fatores sociodemográficos para a diferença entre peso corporal medido e autorreferido. Homens na faixa etária de 30 a 39 anos apresentaram menor especificidade e mulheres acima de 60 anos menor sensibilidade para referir o excesso de peso. O IMC obtido a partir de peso e altura autorreferidos pode ser usado para acompanhamento do excesso de peso.

Efetivamente, a utilização dos dados referidos tem repercussão na economia de recursos financeiros, o que é importante na realização de grandes estudos populacionais, como, por exemplo, o Vigitel, que é conduzido por meio de contato telefônico ${ }^{30}$. A partir dos resultados apresentados, as pesquisas que utilizam o peso e altura em grande número de indivíduos de Brasília podem se beneficiar do uso do peso e altura autorreferidos.

\section{Referências}

1. Wada K, Tamakoshi K, Tsunekawa T, Otsuka R, Zhang $\mathrm{H}$, Murata $\mathrm{C}$ et al. Validity of self-reported height and weight in a Japanese workplace population. Int J Obes 2005; 29(9): 1093-9.

2. Niedhammer I, Bugel I, Bonenfant S, Goldberg M, Leclerc A. Validity of self-reported weight and height in the French GAZEL cohort. Int J Obes Relat Metab Disord 2000; 24(9): 1111-8.

3. Kuczmarski MF, Kuczmarski RJ, Najjar M. Effects of age on validity of self-reported height, weight, and body mass index: findings from the Third National Health and Nutrition Examination Survey, 1988-1994. J Am Diet Assoc 2001; 101(1): 28-34; quiz 35-6.

4. Fonseca MJ, Faerstein E, Chor D, Lopes CS. Validade de peso e estatura informados e índice de massa corporal: estudo pró-saúde. Rev Saúde Pública 2004; 38(3): 392-8.

5. Gorber SC, Tremblay M, Moher D, Gorber B. A comparison of direct vs. self-report measures for assessing height, weight and body mass index: a systematic review. Obes Rev 2007; 8(4): 307-26.

6. Nieto-Garcia FJ, Bush TL, Keyl PM. Body mass definitions of obesity: sensitivity and specificity using self-reported weight and height. Epidemiology 1990; 1(2): 146-52.

7. Alvarez-Torices JC, Franch-Nadal J, Alvarez-Guisasola F, Hernandez-Mejia R, Cueto-Espina RA. Self-reported height and weight and prevalence of obesity: study in a
Spanish population. Int J Obes Relat Metab Disord 1993; 17(11): 663-7.

8. Nakamura K, Hoshino Y, Kodama K, Yamamoto M. Reliability of self-reported body height and weight of adult japanese women. J Biosoc Sci 1999; 31(4): 555-8.

9. Bland JM, Altman DG. Statistical methods for assessing agreement between two methods of clinical measurement. Lancet 1986; 1(8476): 307-10.

10. Schmidt MI, Duncan BB, Tavares M, Polanczyk CA, Pellanda L, Zimmer PM. Validity of self-reported weight-a study of urban Brazilian adults. Rev Saúde Pública 1993; 27(4): 271-6.

11. Villanueva EV. The validity of self-report weight in US adults: a population based cross-sectional study. $B M C$ Public Health 2001; 1: 11.

12. Hill A, Roberts J. Body mass index: a comparison between self-reported and measured height and weight. J Public Health Med 1998; 20(2): 206-10.

13. Peixoto Mdo R, Benício MH, Jardim PC. Validity of selfreported weight and height: the Goiânia study, Brazil. Rev Saúde Pública 2006; 40(6): 1065-72.

14. Nyholm M, Gullberg B, Merlo J, Lundqvist-Persson C, Råstam L, Lindblad U. The validity of obesity based on self-reported weight and height: Implications for population studies. Obesity 2007; 15(1): 197-208. 
15. Silveira EA, Araújo CL, Gigante DP, Barros AJD, Lima MS. Validação do peso e altura Referidos para o diagnóstico do estado nutricional em uma população de adultos no sul do Brasil. Cad Saúde Pública 2005; 21(1): 235-45.

16. Brasil. Governo do Distrito Federal. Portal do Cidadão. Administrações Regionais, Brasília, RA I. Disponível em http://www.brasilia.df.gov.br/ [Acessado em 16 de abril de 2012]

17. Cochran WG. Sampling Techniques. $3^{\text {rd }}$ ed. New York: Wiley; 1977.

18. Craig CL, Marshall AL, Sjöström M, Bauman AE, Booth ML, Ainsworth BE et al. International Physical Activity Questionnaire: 12-Country Reliability and Validity. Med Sci Sports Exerc 2003; 35(8): 1381-95.

19. World Health Organization (WHO). Guide to Physical Measurements (Step 2). WHO STEPS Surveillance; 2008.

20. World Health Organization (WHO). Obesity: preventing and managing the global epidemic. Geneva; 1997.

21. Pate RR, Pratt M, Blair SN, Haskell WL, Macera CA, Bouchard C et al. Physical activity and public health. A recommendation from the Centers for Disease Control and Prevention and the American College of Sports Medicine. JAMA 1995; 273(5): 402-7.

22. Hallal PC, Victora CG, Wells JC, Lima RC. Physical inactivity: prevalence and associated variables in Brazilian adults. Med Sci Sports Exerc 2003; 35(11): 1894900 .

23. Associação Nacional de Empresas de Pesquisa. ANEP. Critério de classificação econômica Brasil. São Paulo; 1996.

24. Ziebland S, Thorogood M, Fuller A, Muir J. Desire for the body normal: body image and discrepancies between self reported and measured height and weight in a British population. J Epidemiol Community Health 1996; 50(1): 105-6.
25. Engstrom JL, Paterson SA, Doherty A, Trabulsi M Speer KL. Accuracy of self-reported height and weight in women: an integrative review of the literature. $J$ Midwifery Women Health 2003; 48(5): 338-45.

26. Brunner Huber LR. Validity of self-reported height and weight in women of reproductive age. Matern Child Health J 2007; 11(2): 137-44.

27. Lim LL, Seubsman SA, Sleigh A. Validity of self-reported weight, height, and body mass index among university students in Thailand: Implications for population studies of obesity in developing countries. Popul Health Metr 2009; 7: 15.

28. Brestoff JR, Perry IJ, Van den Broeck J. Challenging the role of social norms regarding body weight as an explanation for weight, height, and BMI misreporting biases: development and application of a new approach to examining misreporting and misclassification bias in surveys. BMC Public Health 2011; 11: 331.

29. Osuna-Ramírez I, Hernández-Prado B,Campuzano JC, Salmerón J. Body mass index and body image perception in Mexican adult population: The accuracy of self-reporting. Salud Publica Mex 2006; 48: 94-103.

30. BRASIL. Ministério da Saúde. Secretaria de Vigilância em Saúde. Secretaria de Gestão Estratégica e Participativa. Vigitel Brasil 2011: vigilância de fatores de risco $e$ proteção para doenças crônicas por inquérito telefônico. Disponível em http://portalsaude.saude.gov.br/ portalsaude/arquivos/pdf/2012/Abr/10/vigitel_100412. pdf [Acessado em 15 de abril de 2012]

Recebido em: 01/12/10 Versão final apresentada em: 24/04/12 Aprovado em: 27/06/12 